\title{
La formación de la inteligencia colectiva y la Universidad ${ }^{1}$ \\ The formation of collective intelligence and the University \\ A formaçao da inteligência colectiva e a Universidade
}

DOI: http://dx.doi.org/10.25087/resur4a6

Augusto Pérez Lindo ${ }^{2}$

\section{Resumen}

La formación de una inteligencia colectiva tiene que ver con las políticas científicas y universitarias, pero es algo más que eso: es la definición del modelo cultural de desarrollo de un país. Desde mediados de 1970 se vienen formulando en América Latina teorías y programas destinados a aplicar las capacidades universitarias y científicas en la resolución de problemas de la sociedad. Personalidades como Celso Furtado, Amílcar Herrera, Oscar Varsavsky o Jorge Sábato, fueron precursores de un nuevo paradigma que podemos identificar como el Modo 3 de Producción de Conocimientos, es decir, un enfoque que sostiene la necesidad de articular la enseñanza, la investigación y la aplicación de conocimientos útiles al país. Este trabajo presenta las características de este Modo 3, basado en la formación de una inteligencia colectiva en el que cabe un rol clave a la Universidad Sudamericana

Palabras clave: Inteligencia colectiva - Universidad - Modo 3 de Producción de conocimiento

\footnotetext{
${ }^{1}$ Texto basado en la ponencia realizada en el XVII COLOQUIO INTERNACIONAL DE GESTIÓN UNIVERSITARIA Universidad, desarrollo y futuro en la sociedad del conocimiento. Universidad Nacional de Mar del Plata, 22-24 de noviembre de 2017.

2 Doctor en Filosofía. Profesor de diversas universidades argentinas, de la región y del ámbito internacional. Miembro del Consejo Consultivo Asesor de IUSUR. Co-director de RESUR -perezlindo@gmail.com

Rev. educ. super. sur glob - RESUR N4 jul. - dic. 2017 e014 ISSN 2393-6789
} 


\begin{abstract}
The formation of a collective intelligence has to do with scientific and university policies, but it is something more than that: it is the definition of the cultural model of development of a country. Since the mid-1970s, theories and programs designed to apply the university and scientific capacities in the resolution of society's problems have been formulated in Latin America. Personalities such as Celso Furtado, Amílcar Herrera, Oscar Varsavsky and Jorge Sábato, were precursors of a new paradigm that we can identify as Mode 3 of Knowledge Production, that is, an approach that supports the need to articulate teaching, research and education. Application of useful knowledge to the country. This paper presents the characteristics of this Mode 3, based on the formation of a collective intelligence in which South American University plays a key role
\end{abstract}

Keywords: Collective Intelligence - University - Mode 3 of Knowledge Production

\title{
Resumo
}

A formação de uma inteligência coletiva tem a ver com as políticas científicas e universitárias, mas é ainda mais do que isso: é a definição de um modelo cultural de desenvolvimento de um país. Desde mediados de 1970 formulam-se na América Latina teorias e programas, visando aplicar as capacidades universitárias e científicas na resolução de problemas da sociedade. Personalidades como Celso Furtado, Amílcar Herrera, Oscar Varsavsky o Jorge Sábato, foram precursores de um novo paradigma que podemos identificar como o Modo 3 de Produção de Conhecimento, isto é, um enfoque que mantém a necessidade de articular a pesquisa, a pesquisa e a aplicação de conhecimento útil para o país. Este trabalho apresenta as características de este Modo 3, baseado na formação de uma inteligência coletiva na qual tem uma função chave a Universidade Sul Americana.

Palavras-chave: Inteligência coletiva - Universidade - Modo 3 de Produção de conhecimento 
Me propongo en esta ponencia reflexionar sobre la misión de las universidades de América Latina como formadoras de una inteligencia colectiva para crear un modelo de desarrollo inteligente, solidario y sustentable. Si bien, existe un amplio consenso sobre la importancia de los aportes del conocimiento científico y técnico al desarrollo, cabe preguntarse por qué los países de América Latina que poseen un gran capital intelectual con sus universidades y sistemas científicos, no consiguen aprovecharlo para salir del subdesarrollo.

La contracara son los países asiáticos que partiendo de condiciones menos favorables hace cincuenta años revirtieron sus situaciones de atraso y se convirtieron como China o Corea en sociedades avanzadas. En el caso de América Latina lo notable es la falta de un consenso estratégico para lograr resultados semejantes. Quiero indagar sobre la posibilidad de que las universidades de la región contribuyan a crear una inteligencia colectiva que haga posible el surgimiento de un nuevo modelo de desarrollo.

Desde un punto de vista antropológico podemos definir la inteligencia colectiva como una estructura intersubjetiva por la cual se valoriza el conocimiento en tanto herramienta para el progreso y se movilizan las capacidades de los individuos para lograr un desarrollo deseable.

La formación de una inteligencia colectiva tiene que ver con las políticas científicas y universitarias, pero es algo más que eso: es la definición del modelo cultural de desarrollo de un país. Desde mediados de 1970 se vienen formulando en América Latina teorías y programas destinados a aplicar las capacidades universitarias y científicas en la resolución de problemas de la sociedad. Personalidades como Celso Furtado, Amílcar Herrera, Oscar Varsavsky o Jorge Sábato, fueron precursores de un nuevo paradigma que podemos identificar como el Modo 3 de Producción de Conocimientos, es decir, un enfoque que sostiene la necesidad de articular la enseñanza, la investigación y la aplicación de conocimientos útiles al país. Se han realizado muchas experiencias en este sentido, pero no disponemos de un inventario de todas ellas. Tampoco se ha producido un cambio en el modelo de gestión: todavía no tenemos universidades inteligentes. Sigue prevaleciendo la burocracia tradicional, la endogamia académica y la desarticulación entre los productores de conocimiento y la sociedad. 
El filósofo Hegel decía que cuando un pueblo no se reconoce en su experiencia histórica se produce una alienación, o sea, una disociación entre la práctica social y la consciencia sobre la misma. Uno de nuestros males consiste en la desarticulación entre el pensamiento y la acción, entre la ideología y la política real. Es como si padeciéramos un mal metafísico: la separación entre el Ser y el Conocer. En algunos casos convivimos con culturas que rechazan el conocimiento.

El primer paso hacia la formación de una inteligencia colectiva debería ser la toma de conciencia de nuestras propias experiencias. A menudo, en medio de cambios políticos coyunturales muchos piensan que hay que comenzar todo de nuevo. Es la tentación fundacional. Pero en la vida de los pueblos es tan importante cambiar como asimilar las experiencias del pasado. El homo sapiens se distingue como especie porque es capaz de aprender, para eso inventamos la educación y el pensamiento científico.

Tener autoconsciencia, tener consciencia de sí colectivamente es una condición básica para llegar a ser un actor histórico. En este punto es donde las universidades pueden y deben insistir para formar la consciencia colectiva y para orientar a las clases dirigentes. ¿Cuántas de nuestras universidades trabajan en este sentido? Casi todas actúan bajo el supuesto de que nuestra misión se agota en la formación de profesionales o en la organización de algunos proyectos de investigación.

En la actualidad se ha vuelto un lugar común reconocer que las ciencias y las tecnologías están transformando el mundo. No todos tienen en cuenta que el surgimiento de la sociedad del conocimiento depende de otro vértice: el de la educación. Hablamos de la educación en el sentido de la capacidad colectiva para actuar de manera inteligente frente a los problemas que enfrentamos.

Si tenemos en cuenta los criterios anunciados no podemos afirmar que tenemos sociedades inteligentes como tampoco podemos sostener que tenemos Estados inteligentes. Sin embargo, los datos muestran que en América Latina hay más de 25 millones de universitarios y más de 20 millones de personas con educación superior en la fuerza de trabajo. Sin hablar de los intelectuales, científicos y profesionales destacados en todas las ramas del saber. Los especialistas en Ciencia, Tecnología y Sociedad dicen que existe un

Rev. educ. super. sur glob - RESUR No4 jul. - dic. 2017 e014 ISSN 2393-6789 
gran desaprovechamiento de ese potencial. Y una expulsión de recursos altamente calificados que alcanza a más de 300.000 graduados latinoamericanos en otras regiones del mundo. No estamos actuando de manera inteligente y por lo tanto nada asegura que seamos protagonistas de la sociedad del conocimiento sino simples consumidores dependientes de los saberes y poderes que vienen de otras partes.

La teoría de las organizaciones nos enseña que en las instituciones se producen "culturas organizacionales" que determinan el sentido de sus actividades. La "identidad institucional" resume un conjunto de prácticas pedagógicas y de gestión que facilitan el logro de los propósitos colectivos. Con bastante optimismo supuse durante décadas que las universidades argentinas y latinoamericanas podían crear una nueva "cultura organizacional" convirtiéndose en agentes de desarrollo mediante políticas de conocimiento. Desde esa perspectiva me parecía inevitable la aceptación de la idea de una universidad como agente de desarrollo. Pero si bien el concepto ha sido aceptado entre los especialistas y dirigentes universitarios todavía no forma parte del consenso social.

Cuando uno revisa la función social del conocimiento en una perspectiva evolucionista se da cuenta que desde los albores de la historización humana los grupos de homo sapiens pudieron evolucionar y progresar porque empezaron a acumular conocimientos, crearon formas de organización y de división del trabajo, desarrollaron el lenguaje y adoptaron principios de ensayo y error para adaptarse al medio y descubrir nuevas posibilidades de vida. Lo más importante, a mi entender, es que el homo sapiens fue creando formas de inteligencia colectiva que diferenciaron la trayectoria de las comunidades exitosas.

Durante el siglo XX la inteligencia colectiva estuvo asociada a la consolidación de los Estados - nacionales. En la actualidad, con la globalización y la informatización de las sociedades surgen nuevas formas de inteligencia colectiva porque el espacio del conocimiento se vuelve ubicuo y multidimensional. Estados, corporaciones, organizaciones sociales, todos tratan de reubicarse frente al nuevo contexto de la sociedad del conocimiento. Surgen redes sociales de todo tipo compitiendo para formar nuevas consciencias colectivas a través de Internet. 
Resulta importante señalar que de acuerdo con Michael Gibbons y otros expertos asistimos al nacimiento de un nuevo modo de producción y distribución de conocimientos. Vamos hacia una cultura de conocimiento distribuido. La expansión de Internet, de las redes sociales y de los sistemas de información permiten que surjan inteligencias colectivas en muchas dimensiones, fuera de los circuitos formales de la educación y la investigación académica. Esto señala también la responsabilidad de las universidades para conservar la cultura del conocimiento científico en la era de Internet.

En este escenario nos preguntamos de qué manera las universidades sudamericanas podrían contribuir a crear una inteligencia colectiva que permita aprovechar los conocimientos disponibles en la era informática y global, para crear un proceso de desarrollo virtuoso.

Si analizamos las experiencias internacionales de los últimos cincuenta años podemos identificar distintos casos de reelaboración de la inteligencia colectiva en Corea del Sur, Israel, la Unión Europea, China, Rusia, Sudáfrica, Finlandia, Corea del Norte, Irán, los Emiratos Árabes. Estos y otros casos muestran como en contextos muy diversos se pueden crear capacidades para movilizar las competencias de los individuos a fin de lograr objetivos socialmente compartidos.

En Europa a partir del Acuerdo de Bolonia en 1999 un grupo de universidades propuso crear un Espacio Europeo del Conocimiento a fin de competir con Estados Unidos y Japón. El Proceso de Bolonia hoy involucra universidades de 54 países, muchos ajenos a la Unión Europea. Esto constituye el mayor ejemplo de un intento para plasmar la integración de sociedades y culturas diferentes en busca de un desarrollo común contando con el potencial tecnológico y científico. Esta arquitectura supone un pensamiento complejo, una gran flexibilidad y una gran capacidad para administrar situaciones a veces contradictorias.

Si nos situamos en América Latina podemos constatar que se han intentado diversos formatos para lograr la integración regional: la ALALC, el Pacto Andino, el Mercosur, la Alianza del Pacífico, el Mercado Centroamericano, la CELAC, el UNASUR y otros. Todos estos proyectos quedaron a mitad de camino y algunos están en proceso de reestructuración. La mayoría de estos intentos se centraron o bien en la creación de un mercado regional o

Rev. educ. super. sur glob - RESUR No4 jul. - dic. 2017 e014 ISSN 2393-6789 
bien en un proyecto de integración política. Ninguno intentó crear un proceso de desarrollo inteligente con uso intensivo del conocimiento.

Debemos destacar el caso del Ecuador que desde la Constitución de 2008 se propuso fortalecer las universidades y centros científicos para promover la sociedad del conocimiento. Se invirtió el 2\% del PBI en Educación Superior y actividad científica. Se crearon sistemas de becas para formar profesores en el exterior. El Gobierno asumió el liderazgo de este proceso. En otros países de América Latina mejoró el financiamiento científico y universitario, se crearon programas de extensión y transferencia hacia la sociedad y las empresas. Pero nadie se propuso colocar a las universidades como agentes centrales de un proyecto de desarrollo.

La cuestión es saber: ¿por qué habrían de estar las universidades y los centros científicos en el centro de la escena? Hacia comienzos del siglo XIX en plena Revolución Industrial en Europa dos pensadores bien distintos como Augusto Comte, creador del Positivismo, y Karl Marx, teórico del comunismo, llegaron a la conclusión de que los técnicos y los obreros industriales estaban produciendo una nueva sociedad y que por lo tanto debían asumirse como protagonistas principales. Max Weber dijo más tarde algo semejante respecto a los burócratas de la administración pública. En la actualidad, en términos cuantitativos y cualitativos los productores de conocimientos tienen más relevancia que la que pensaban esos pensadores. Peter Drucker en un ensayo de 1990 afirmó que ahora los trabajadores calificados, los tecnólogos y los profesionales representan un nuevo proletariado, el cognitariado. La "centralidad" del cognitariado en el mundo actual proviene del hecho de que constituye la base de la producción de la sociedad. Pensemos simplemente qué pasaría si en este momento todos los informáticos del mundo decidieran paralizar sus actividades.

En términos globales América Latina tiene más de 25 millones de estudiantes universitarios y más del 20\% de la población activa con educación superior. Argentina tiene cerca de 2,8 millones de alumnos en la Educación Superior y Brasil más de 8 millones de universitarios. Las capacidades educativas, científicas, universitarias y técnicas de América Latina son abundantes. Sin embargo, muchos siguen pensando el futuro en función de las materias

Rev. educ. super. sur glob - RESUR No4 jul. - dic. 2017 e014 ISSN 2393-6789 
primas: petróleo, soja, cobre, frutos, gas. Pero el futuro de América Latina depende hoy del aprovechamiento de su capital intelectual y social.

Tenemos los recursos humanos. Tenemos los recursos naturales suficientes. ¿Qué falta? Una inteligencia colectiva para aprovechar estas potencialidades y superar el subdesarrollo. Pero, ¿quién lidera este proceso? ¿El Estado?, ¿un Partido?, un líder providencial? Las experiencias muestran que no basta con el liderazgo centralizado en el Estado o el Gobierno. Es necesario que la sociedad asuma una consciencia colectiva de sus posibilidades. Como ocurrió en Japón a fines del siglo XIX, como ocurrió en China a partir de 1977 luego del fracaso de la Revolución Cultural.

En la Alemania de 1809, que se encontraba atrasada en la Revolución Industrial que lideraban Inglaterra y Francia, fue un grupo de filósofos y científicos el que rediseñó la Universidad de Berlín para que sirviera con su producción científica como catapulta del poderío industrial que se logró en las décadas siguientes. Pero la sociedad civil, la burocracia estatal, los empresarios y el poder militar comprendieron el desafío y lo apoyaron.

La sociedad civil en nuestros países todavía no tiene consciencia de lo que significa crear un Estado inteligente y una economía del conocimiento. Entre otras cosas porque vivimos en sociedades fragmentadas con sectores que viven como en el primer mundo y sectores que viven en el cuarto mundo. Prevalecen arcaicas relaciones de dominación, el clientelismo político o la exclusión social. ¿Cómo se puede construir entonces la idea de una sociedad racionalmente organizada? Las estadísticas espantan: Tenemos los índices de desigualdad y de homicidios más altos del mundo. La mayoría de la gente vive en culturas de supervivencia, en economías marginales, en medio de mecanismos irracionales o situaciones dramáticas. Pero las tasas de beneficios de los bancos y las corporaciones nacionales y extranjeras son mayores que en los países ricos.

Las universidades constituyen espacios donde se puede investigar, pensar, estudiar las condiciones de la sociedad, diseñar proyectos, realizar innovaciones. Además, ya han demostrado que tienen capacidades para apoyar emprendimientos económicos, para producir innovaciones tecnológicas, para asesorar a organizaciones sociales y estatales en

Rev. educ. super. sur glob - RESUR No4 jul. - dic. 2017 e014 ISSN 2393-6789 
diversos proyectos. Las universidades poseen un capital intelectual enorme pero no tienen consciencia de su potencial. Siguen creyendo que su función dominante es la de formar profesionales. No se dan cuenta que ahora lo importante no es tener profesionales sino saber aprovecharlos.

A las universidades incumbe pensar el futuro de sus graduados, su empleabilidad, su aprovechamiento por la sociedad, la economía y el Estado. Esta es ahora su misión histórica. Por eso debe asumir el lugar de una inteligencia colectiva y proponerse además crear en la sociedad la consciencia del potencial que representan los recursos científicos, profesionales, educativos del país.

Ante todo, deberíamos realizar un inventario objetivo del capital intelectual de los países de América Latina que va más allá del número de estudiantes, de profesores, de profesionales y científicos. Hay que considerar, entre otros aspectos, el potencial que representan miles de organizaciones sociales capaces de crear innovaciones, emprendimientos y formas de solidaridad. El capital intelectual no debe disociarse del capital social.

La cuestión fundamental es la del consenso estratégico entre las universidades para crear alternativas inteligentes, solidarias y sustentables de desarrollo. Las universidades de Argentina y Brasil han creado incubadoras de empresas y centros tecnológicos. En Brasil se han diseñado varias ciudades inteligentes. Se ha demostrado la capacidad para apoyar emprendimientos sociales, empresariales y estatales. Las políticas de vinculación con la sociedad, las empresas o el Estado han ganado mucho terreno. Pero la mayoría sigue pensando que a las universidades les incumbe investigar y formar profesionales, no ocuparse del desarrollo del país.'

Otro obstáculo importante: la fragmentación de las universidades, al interior de sí mismas y en el sistema de educación superior de cada país. El individualismo institucional lleva a veces a la feudalización de cátedras, departamentos, institutos, carreras, facultades. Y a esto hay que agregar la ausencia de políticas nacionales para articular las instituciones de educación superior entre sí, con la sociedad, con la economía, con el Estado.

Generalmente se invoca la autonomía universitaria o las peculiaridades de cada institución para justificar la atomización del sistema. La experiencia del proceso de Bolonia en Europa 
muestra que las universidades pueden aprovechar la autonomía para crear convergencias y formas de cooperación entre 54 países de Europa y África con identidades muy diversas. La política del conocimiento al servicio del desarrollo común se puso por encima de lenguas, identidades, conflictos seculares e intereses particulares. Con muchas menos barreras culturales las universidades de América Latina podrían converger autónomamente en torno a un proceso cooperativo. Las diversidades políticas y económicas no constituyen una barrera insalvable para avanzar hacia un proyecto común.

Hemos avanzado mucho en la formación de recursos humanos de alto nivel y en el diseño de investigaciones avanzadas en todos los campos. Se ha progresado en la introducción de políticas de extensión, de transferencia tecnológica, de voluntariado social en casi todas las universidades. Pero nos falta crear consciencia de la posibilidad de aprovechar nuestro capital intelectual para iniciar una nueva era de desarrollo inteligente.

Todavía no tenemos el suficiente acuerdo de las clases dirigentes en la sociedad civil para crear modos de articulación exitosos entre las universidades, los investigadores, las empresas, las organizaciones y el Estado. En esto podrían jugar un rol fundamental los medios de comunicación que raramente se preocupan por la cultura científica, tecnológica o universitaria. Deberíamos pensar estrategias para lograr a través de los medios de comunicación y las redes sociales la instalación pública de un proyecto de desarrollo inteligente y solidario. Los medios deberían contar como aliados de este proceso.

¿Pueden crear las universidades liderar un proceso para facilitar el surgimiento de un desarrollo inteligente, solidario y sustentable? Por supuesto que pueden, porque la experiencia histórica muestra ejemplos en ese sentido. En medio de crisis económicas y de fragmentaciones políticas o sociales pareciera que las clases dirigentes no están en condiciones de liderar un proceso semejante. Las universidades pueden situarse más allá de las coyunturas y mirar el futuro para trascender los conflictos del momento en función de un proyecto histórico más trascendente. Obviamente, se necesitan núcleos y liderazgos institucionales convergentes con la formación de una nueva inteligencia colectiva.

Como primer paso hacia la construcción de la nueva inteligencia colectiva las universidades latinoamericanas podrían auto convocarse en un Foro Regional para discutir las alternativas

Rev. educ. super. sur glob - RESUR No4 jul. - dic. 2017 e014 ISSN 2393-6789 
para un desarrollo inteligente, solidario y sustentable en América Latina. De ese Foro deberían surgir proyectos concretos para convocar a todos los sectores sociales a fin de discutir las estrategias más convenientes para implementarlos. Para ampliar el consenso estratégico deberíamos intercambiar informaciones sobre las experiencias exitosas de las universidades latinoamericanas en proyectos de desarrollo local y regional. El "efecto demostración” es el mejor argumento para derribar al pesimismo.

América Latina tiene todas las posibilidades para construir sociedades prósperas y justas, pero carece de una intencionalidad pragmática para lograrlo. Sería una misión histórica de las universidades crear una inteligencia colectiva que nos permita llegar a ser lo que podemos ser. Tenemos que hacernos cargo. Las universidades latinoamericanas deben tener la lucidez y la audacia para valorar el conocimiento distribuido en nuestras sociedades a fin de aprovechar todas las potencialidades para lograr el desarrollo colectivo y la realización de las personas.

Rev. educ. super. sur glob - RESUR No4 jul. - dic. 2017 e014 ISSN 2393-6789 